\title{
Alx3-deficient mice exhibit decreased insulin in beta cells, altered glucose homeostasis and increased apoptosis in pancreatic islets
}

\author{
M. Mirasierra • A. Fernández-Pérez • N. Díaz-Prieto • \\ M. Vallejo
}

Received: 26 April 2010 / Accepted: 20 October 2010 /Published online: 21 November 2010

(C) Springer-Verlag 2010

\begin{abstract}
Aims/hypothesis Homeodomain transcription factors play an important role in the regulation of pancreatic islet function. In previous studies we determined that aristaless-like homeobox 3 (ALX3) is produced in islet cells, binds to the promoter of the insulin gene and regulates its expression. The purpose of the present study was to investigate the functional role of ALX3 in pancreatic islets and its possible involvement in the regulation of glucose homeostasis in vivo.

Methods Alx3-knockout mice were used. Glucose and insulin tolerance tests were carried out, and serum insulin concentrations were determined. Isolated islets were used to test insulin secretion and gene expression. The pancreatic islets were also studied using both confocal and conventional microscopy.

Results ALX3 deficiency resulted in increased blood glucose levels and impaired glucose tolerance in the presence of normal serum insulin concentrations. Insulin, glucagon and glucokinase expression were reduced in Alx3null pancreatic islets. Reduced insulin content was reflected by decreased insulin secretion from isolated islets. Alx3-
\end{abstract}

Electronic supplementary material The online version of this article (doi:10.1007/s00125-010-1975-6) contains supplementary material, which is available to authorised users.

M. Mirasierra • A. Fernández-Pérez • N. Díaz-Prieto •

M. Vallejo $(\square)$

Centro de Investigación Biomédica en Red de Diabetes y

Enfermedades Metabólicas Asociadas (CIBERDEM),

Instituto de Investigaciones Biomédicas 'Alberto Sols',

Consejo Superior de Investigaciones Científicas

/Universidad Autónoma de Madrid,

Calle Arturo Duperier 4,

28029, Madrid, Spain

e-mail: mvallejo@iib.uam.es deficient islets also showed increased apoptosis, and morphometric analyses indicated that they were, on average, of smaller size than islets from control mice. ALX3 deficiency resulted in reduced beta cell mass. Finally, mature Alx3-null mice developed age-dependent insulin resistance due to impaired peripheral insulin receptor signalling.

Conclusions/interpretation ALX3 participates in the regulation of the expression of essential genes for the function of pancreatic islets, and its deficiency alters the regulation of glucose homeostasis in vivo. We suggest that ALX3 constitutes a potential candidate to consider in the aetiopathogenesis of diabetes mellitus.

Keywords Apoptosis · Aristaless · Glucagon ·

Glucokinase $\cdot$ Homeodomain $\cdot$ Insulin resistance $\cdot$ Insulin secretion - Transcription

$\begin{array}{ll}\text { Abbreviations } \\ \text { AKT } & \text { Anti-thymoma viral proto-oncogene 1 } \\ \text { ALX3 } & \text { Aristaless-like homeobox } 3 \\ \text { ChIP } & \text { Chromatin immunoprecipitation } \\ \text { GCK } & \text { Glucokinase } \\ \text { HBSS } & \text { Hanks' balanced salt solution } \\ \text { Ki67 } & \text { Antigen identified by monoclonal antibody Ki } 67 \\ \text { PDX1 } & \text { Pancreatic and duodenal homeobox 1 }\end{array}$

\section{Introduction}

Increased death of insulin-producing beta cells in pancreatic islets leading to insulin deficiency and hyperglycaemia constitutes a hallmark of diabetes mellitus. Animal studies have identified a number of defects associated with 
increased apoptosis of beta cells, including endoplasmic reticulum stress [1], defects in intracellular signalling pathways [2] and mitochondrial dysfunction [3]. The isolated deficiency of some transcription factors that regulate islet cell development and function also produce beta cell apoptosis [4]. Prominent among these are BETA2 (also known as neurogenic differentiation 1 [NEUROD1]) and pancreatic and duodenal homeobox 1 (PDX1), two important regulators of insulin gene expression. In the first case, mice deficient in Beta2 (also known as Neurod1) exhibit massive death of newly formed beta cells [5]. In the second case, beta cell apoptosis is due to $P d x 1$ haploinsufficiency in heterozygote animals [6]. In addition, conditional inactivation of $I s l l$ has revealed increased postnatal islet cell death and diabetes [7]. Finally, loss of function of another important transcription factor, hepatocyte nuclear factor $1-\alpha(\mathrm{HNF} 1 \alpha)$, can induce mitochondrial dysfunction and islet cell apoptosis [8].

The importance of these findings is underscored by the observations that, in humans, mutations in the genes that encode these transcription factors have been associated with different forms of diabetes. Thus, mutations in BETA2 are associated with MODY6 [9]; mutations in PDX1 are associated with MODY4 and with late-onset type 2 diabetes $[10,11]$; mutations in $I S L 1$ can be associated with type 2 diabetes [12, 13]; and mutations in $H N F 1 A$ are causative of MODY3, the most common type of monogenic diabetes [14].

Some of these transcription factors regulate complex transcriptional programmes of gene expression by acting on pancreatic cell specific target gene networks $[15,16]$. Furthermore, in combination with other transcription factors present in mature cells, they interact co-ordinately on the insulin gene promoter to regulate insulin gene expression [4, 17]. Thus, the integrity of transcription factors involved in the regulation of insulin gene expression appears to be important for preventing islet beta cell dysfunction leading to diabetes.

Previous studies have indicated that aristaless-like homeobox 3 (ALX3) [18] is produced in glucagon-, somatostatinand insulin-producing cells of pancreatic islets [19]. As those studies identified the insulin gene as the first known target for regulation by ALX3, it became important to investigate the role of ALX3 in pancreatic beta cell function in vivo. In the present study, we show that ALX3 deficiency in mice is associated with impaired glucose homeostasis and with increased cell death in pancreatic islets.

\section{Methods}

Alx3-deficient mice Mice deficient in Alx3, generated by homologous recombination, were provided by F. Meijlink
(Netherlands Institute for Developmental Biology, Utrecht, the Netherlands) [20]. Genotyping was performed by PCR as described by Beverdam et al. [20], with the exception that the sequence of the forward primer for the wild-type allele was 5'-CATCCCCTCTCCATGCATGTCCCC-3'. Experiments were performed with male 12-16-week-old mice, unless indicated otherwise. Experimental protocols involving mice were approved by the institutional bioethics committee on research animal care, and met the requirements of Spanish and European Community legislation.

Blood glucose and insulin determinations Glucose levels were measured from blood obtained from the tail vein after an overnight fasting period using an automated glucometer (Glucotrend Soft Test System; Boehringer Ingelheim, Mannheim, Germany). For glucose tolerance tests, after measuring baseline glucose levels, mice were injected intraperitoneally with glucose $(2 \mathrm{~g} / \mathrm{kg})$, and blood was tested 15, 60 and 120 min after the injection.

For insulin tolerance tests, food was removed $4 \mathrm{~h}$ before the test. Basal blood glucose concentrations were measured, and then insulin (Actrapid; Novo Nordisk, Bagsvaert, Denmark) was injected intraperitoneally at a dose of $0.75 \mathrm{U} / \mathrm{kg}$. Glucose concentrations were measured again at the indicated times after insulin administration. Serum insulin was measured using an ELISA kit (Crystal Chem, Downers Grove, IL, USA).

Immunofluorescence Pancreases were fixed with 4\% paraformaldehyde and cryostat sections $(10 \mu \mathrm{m})$ were cut. The primary antibodies used are indicated in Electronic supplementary material (ESM) Table 1. Secondary antibodies were Texas Red anti-guinea pig IgG (Vector Laboratories, Burlingame, CA, USA; dilution 1:100), 546-Alexa or 488-Alexa anti-rabbit (Invitrogen, Carlsbad, CA, USA; 1:500 dilution). A minimum of 16-20 sections per pancreas from at least four animals of each genotype were analysed.

TUNEL assays A commercial ApopTag Plus Peroxidase In Situ Apoptosis Detection kit (Millipore, Billerica, MA, USA) was used. Immunodetection was performed using diaminobenzidine staining. The number of immunopositive cells in each islet present in sections separated by at least $80 \mu \mathrm{m}$ was counted from digital images using NIH ImageJ software. For newborn mice, the entire digestive tract including the pancreas was dissected and cryostat sections $(10 \mu \mathrm{m})$ were cut. Apoptotic cells were detected using diaminobenzidine staining enhanced with nickel ammonium (dark grey colour). Sections were then washed and standard insulin immunoperoxidase staining was performed (brown colour). 
Isolation of pancreatic islets The pancreas was inflated by injecting $3 \mathrm{ml}$ of HEPES (10 mmol/l)/Hanks' balanced salt solution (HBSS) buffer containing collagenase NB8 (1.36 U/ml) (SERVA Electrophoresis, Heidelberg, Germany) through the bile duct using a 30 gauge needle. The pancreas was then removed and digested in collagenase solution for $12 \mathrm{~min}$ at $37^{\circ} \mathrm{C}$. After washing in cold HBSS containing $0.5 \%$ (wt/vol.) BSA, islets were purified on a discontinuous gradient formed by a mixture of Histopaque 1077 and Histopaque 1119 (Sigma, Madrid, Spain) (7:3 ratio) on the lower phase, and HBSS-0.5\% (wt/vol.) BSA on the upper phase. The islet-enriched interphase was aspirated, washed, and individual islets were picked using a micropipette under a stereomicroscope.

Glucose-stimulated insulin secretion Batches of ten isolated islets of approximately similar size were transferred to incubation vials containing pre-gassed $\left(95 \% \mathrm{O}_{2} / 5 \% \mathrm{CO}_{2}\right)$ Krebs-Ringer HEPES bicarbonate buffer and $0.5 \%$ (wt/vol.) BSA $(100 \mu \mathrm{l} /$ islet $)$, in the presence of $2.8 \mathrm{mmol} / 1$ glucose. After incubation for $1 \mathrm{~h}$ at $37^{\circ} \mathrm{C}$, batch triplicates (i.e. three tubes containing ten islets each) were transferred to a similar solution containing either $2.8 \mathrm{mmol} / \mathrm{l}$ or $20 \mathrm{mmol} / \mathrm{l}$ glucose. After a second $1 \mathrm{~h}$ incubation period, supernatant fractions were collected and islets were sonicated in acid-ethanol. Insulin was assayed by radioimmunoassay (RI-13 K, Millipore).

Liver and muscle extracts Alx3-deficient mice (32-33 week old) were subjected to insulin tolerance tests to identify insulin-resistant animals. Several days later, mice were treated intraperitoneally with insulin $(0.75 \mathrm{U} / \mathrm{kg})$, and after 15 min they were killed. A fragment of liver and soleus muscle were immediately frozen in liquid nitrogen, and then homogenised in ice-cold lysis buffer [21]. Extracts were centrifuged at $15,000 \mathrm{~g}$ for $40 \mathrm{~min}$ at $4^{\circ} \mathrm{C}$, and the supernatant fractions were collected and stored at $-80^{\circ} \mathrm{C}$.

Western immunoblots Islet lysates were resolved by SDSPAGE and blotted onto a nitrocellulose membrane. The following primary antisera were used: rabbit antiglucokinase (GCK) (ab37796; Abcam, Cambridge, MA, USA, 1:500 dilution); rabbit anti-PDX1 C-terminus (provided by J. Habener, Massachusetts General Hospital, Boston, MA, USA; 1:500 dilution); rabbit anti-histone H3 (ab1791, Abcam; 1:5,000 dilution); and mouse anti-actin monoclonal antibody (clone AC-15, Sigma; 1:10,000 dilution).

Liver and muscle extracts were resolved by SDS-PAGE and blotted onto BioTrace PVDF membranes (Pall Corporation, Pensacola, FL, USA). Membranes were incubated with anti-thymoma viral proto-oncogene 1 (AKT [also known as AKT1]) [21] or anti-phospho-AKT (Ser473; sc-
7985; Santa Cruz Biotechnology, Santa Cruz, CA, USA) primary antibodies.

Secondary antibodies used were goat anti-rabbit or goat anti-mouse peroxidase-conjugated (1:5,000 dilution) (BioRad, Hercules, CA, USA). Immunoreactive bands were visualised by enhanced chemiluminescence (Immobilon Western, Millipore). Films were scanned and densitometry measurements of bands were performed using NIH ImageJ 1.37b software (http://rsbweb.nih.gov/ij/).

Quantitative RT-PCR Total RNA from isolated islets was extracted using the Illustra RNAspin kit (GE Healthcare Europe, Barcelona, Spain). Quantitative PCR for Glut2, Gck, $P d x 1$ and glucagon was performed with TaqMan Assay-on-Demand primers and the Taqman Universal PCR Master Mix, No AmpErase UNG (Applied Biosystems, Alcobendas, Madrid, Spain). For insulin, somatostatin and Irs2, SYBR Green detection was used with Power SYBR Green PCR Master Mix (Applied Biosystems) and the primers indicated in ESM Table 2. PCR reactions were performed in triplicate in a $7900 \mathrm{HT}$ fast real-time PCR system (Applied Biosystems), and values were normalised to Gapdh mRNA levels.

Chromatin immunoprecipitation assays Two independent chromatin immunoprecipitation (ChIP) assays were performed on mouse islets as described by Mirasierra and Vallejo [19]. PCR was performed using oligonucleotide primers that amplify a fragment of the Gck (nucleotides -256 to -1 ) [22], or glucagon (nucleotides -353 to +7 ) [23] genes (ESM Table 3). PCR conditions were as follows: $95^{\circ} \mathrm{C}$ for $5 \mathrm{~min}$, followed by 30 cycles of $94^{\circ} \mathrm{C}$ for $30 \mathrm{~s}, 61^{\circ} \mathrm{C}$ for $30 \mathrm{~s}$, and $72^{\circ} \mathrm{C}$ for $30 \mathrm{~s}$, after which there was $5 \mathrm{~min}$ incubation at $72^{\circ} \mathrm{C}$. As a control, we used promoter sequences from the Pck gene as described [19]. A third independent ChIP assay was performed and analysed by quantitative PCR in triplicate samples. In this case the same PCR primers for Gck and Pck were used.

Morphometric analysis Cryostat sections $(10 \mu \mathrm{m})$ of pancreases were stained with Cresyl Violet and photographed. The number of islets present in sections separated by at least $80 \mu \mathrm{m}$ were counted, and the area of each islet was measured using NIH ImageJ $1.37 \mathrm{~b}$ software. For the determination of beta cell mass, the whole pancreases from three mice of each genotype were removed, weighed, fixed in $4 \%$ paraformaldehyde and embedded in paraffin. Longitudinal sections of $10 \mu \mathrm{m}$ thickness generated every $100 \mu \mathrm{m}$ were serially processed for diaminobenzidine immunohistochemistry using guinea pig anti-human insulin (Linco Research, St Charles, MO, USA; 1:100 dilution). Sections were counterstained with haematoxylin and photographed on a Nikon 90i microscope. The area of the 
insulin-stained cells as well as the entire area of pancreatic tissue in each section was calculated on calibrated digital images using NIH ImageJ software. Beta cell mass was then calculated as the product of pancreatic weight and the fractional beta cell area in different sections. A total of 26-29 sections per group were analysed.

\section{Results}

Alx3-deficient mice show impaired glucose homeostasis The body weight curves of Alx3-null and wild-type mice were similar (Fig. 1a), but blood glucose levels in fasting animals were higher in Alx3-deficient mice (Fig. 1b). Importantly, we observed that glucose tolerance is impaired in these mice (Fig. 1c-d). In heterozygote mice, fasting blood glucose levels $(7.31 \pm 0.13 \mathrm{mmol} / \mathrm{l} ; n=6)$ were also significantly higher than in control animals $(p<0.005$, Student's $t$ test). On glucose tolerance testing, the hyperglycaemic pattern exhibited by heterozygote mice was indistinguishable from that observed in homozygote Alx3- deficient animals (not shown), indicating impaired pancreatic islet function due to Alx3 haplo-insufficiency. Fasting glycaemia was higher in mutant females relative to controls $(4.21 \pm 0.29 \mathrm{mmol} / 1$ vs $3.19 \pm 0.25 \mathrm{mmol} / 1, p<0.05$, Student's $t$ test, $n=30$ per group). Alx3-deficient females also exhibited impaired glucose tolerance (not shown). Serum insulin levels in basal conditions or after an injection of glucose were similar in wild-type and Alx3mutant mice, despite the observed differences in glucose levels (Fig. 1e-f). These experiments suggest that the amount of insulin released in Alx3-deficient mice is not sufficient to lower blood glucose levels to values similar to those found in wild-type animals.

We evaluated glucose-stimulated insulin secretion from isolated islets in vitro. Exposure of islets to $20 \mathrm{mmol} / \mathrm{l}$ glucose resulted in insulin secretion in wild-type and mutant mice. However, the values observed in samples corresponding to Alx3-null mice were significantly lower than those found in samples from wild-type animals (Fig. 2a). Measurement of the total insulin content revealed that the amount of hormone in islets was lower in Alx3-deficient mice (Fig. 2b). When

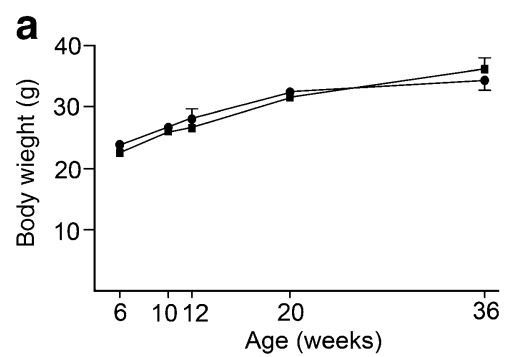

b

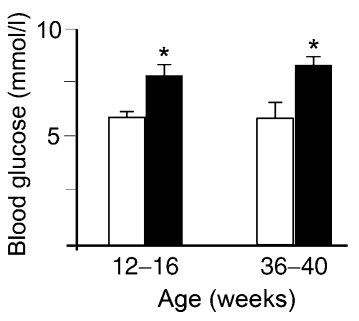

C

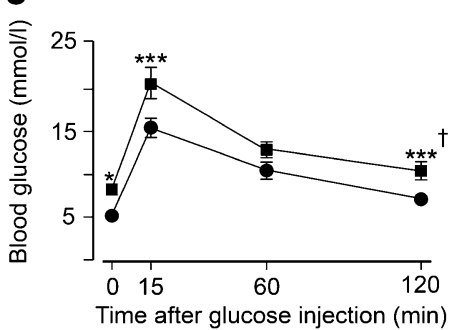

\section{e}

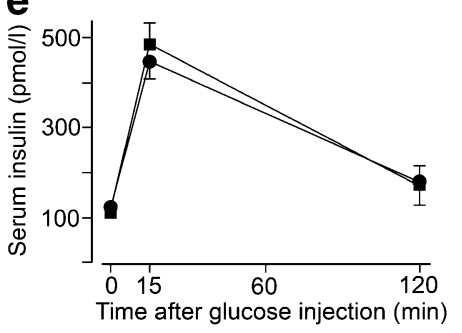

d
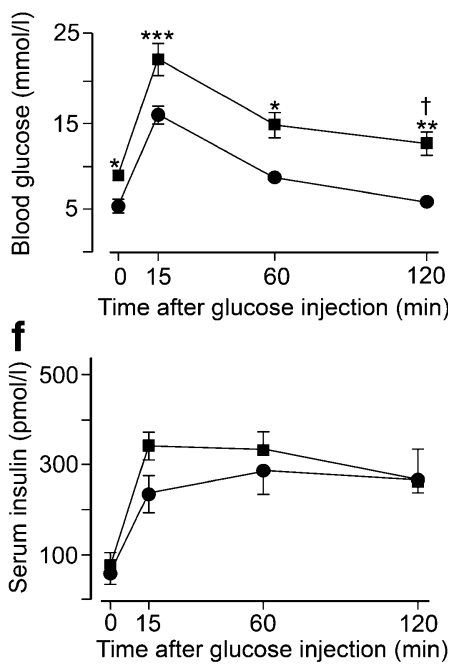

Fig. 1 Mild hyperglycaemia and glucose intolerance in Alx3-deficient mice. a Growth rate in terms of body weight of Alx3-deficient and control wild-type mice monitored between 6 and 36 weeks of life. Data represent the mean \pm SEM of 20-34 animals in each group. Circles, wild-type mice; squares, Alx3-deficient mice. b Basal blood glucose concentrations after fasting in wild-type and Alx3-deficient mice. Data from two different age groups are shown. Each bar represents the mean \pm SEM of determinations taken from 8-14 animals. White bars, wild-type mice; black bars, Alx3-deficient mice. ${ }^{*} p<0.02$, Student's $t$ test. c, d Glucose tolerance tests carried out in fasting wild-type (circles) or Alx3-deficient (squares) mice that received an intraperitoneal injection of glucose $(2 \mathrm{~g} / \mathrm{kg}$ body weight). Data from animals of 12-16 weeks of age (c; $n=9$ and $n=5$, respectively) or 36-40 weeks of age (d; $n=8$ and $n=14$, respectively) are shown. Note that blood glucose concentrations were significantly higher in mutant than in control animals at all times measured, and remained elevated relative to their own basal values for more than $2 \mathrm{~h}$. ${ }^{*} p<0.05, * * p<0.01$ and $* * * p<0.001$ compared with wild-type mice (ANOVA); ${ }^{\dagger} p<0.05$ compared with basal levels before the glucose injection (Student's $t$ test). e, f Serum concentrations of insulin in fasting wild-type (circles) or Alx3-deficient (squares) mice at the indicated times after receiving an intraperitoneal injection of glucose ( $2 \mathrm{~g} / \mathrm{kg}$ body weight). Data from mice of $12-16$ weeks of age (e; $n=6$ in each genotype group) or 36-40 weeks of age (f; $n=5$ and $n=6$, respectively) are shown. In all cases, data represent the mean \pm SEM 


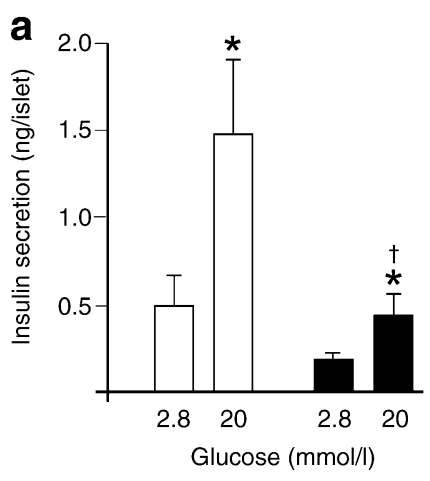

Fig. 2 Glucose-stimulated insulin secretion and insulin content. a Glucose-stimulated insulin secretion from isolated pancreatic islets in vitro. Data are expressed as mean \pm SEM of values obtained from experiments carried out in triplicate. Each animal provided islets for one low-glucose $(2.8 \mathrm{mmol} / \mathrm{l})$ and one high-glucose $(20 \mathrm{mmol} / \mathrm{l})$ triplicate ( $n=6$ for each condition in the case of wild-type islets, and nine for each condition in the case of Alx3-null islets). ${ }^{*} p<0.05$ relative to low-glucose control; ${ }^{\dagger} p<0.01$ relative to wild-type highglucose values, Student's $t$ test. b Insulin content in isolated islets b

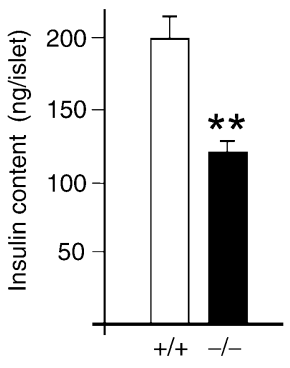

C

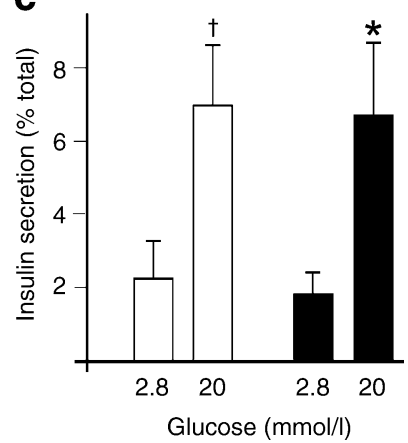

from wild-type $(+/+)$ and Alx3-deficient $(-/-)$ mice. Data are expressed as mean \pm SEM of values obtained from sample tubes (42 for wild-type and 62 for mutant islets), each containing ten islets. $*^{* *} p<0.01$, Student's $t$ test. c Glucose-stimulated insulin secretion from isolated pancreatic islets in vitro. The graph corresponds to data shown in a, but normalised to total insulin content in islets. ${ }^{\dagger} p<0.02$, ${ }^{*} p<0.05$, relative to low-glucose control, Student's $t$ test. White bars, wild-type mice; black bars, Alx3-deficient mice

insulin secretion values were normalised against total insulin content, the percentage of insulin secreted from mutant and wild-type islets was found to be equivalent (Fig. 2c). These data are consistent with the notion that Alx 3 deficiency does not affect the mechanisms involved in glucose-stimulated insulin secretion per se. Rather, decreased secreted insulin appears to be a reflection of decreased insulin content.

Altered gene expression and increased apoptosis in Alx3deficient islets To investigate possible alterations in pancreatic islets, we determined the mRNA levels of Glut2 and $G c k$, as they constitute the first steps in glucose intake and sensing by beta cells. We detected a significant reduction in the levels of Gck mRNA in Alx3-deficient islets (Fig. 3a). This reduction was correlated with a decrease in islet GCK at the protein level, as assessed both by western immunoblot (Fig. 3b, c) and by immunofluorescence (Fig. 3d). In contrast, we found that mRNA levels for Glut2 in islets were similar in Alx3-deficient and in wild-type mice (Fig. 3a). Immunofluorescence staining (Fig. 3d) and western immunoblot (data not shown) confirmed similar levels of expression in both groups. Furthermore, ChIP assays revealed that in pancreatic islets of wild-type animals, ALX3 binds directly to the Gck gene promoter (Fig. 3e-f).

Insulin I and glucagon mRNA levels were also decreased (Fig. 3a). Decreased insulin levels were also observed by immunofluorescence (Fig. 3g) and confirmed by western immunoblot (Fig. 3h). By ChIP assay we found that ALX3 occupies the glucagon gene promoter in vivo

(Fig. 3e). These results were validated by promoter reporter assays in transfected cells (ESM Fig. 1). Together, our data indicate that ALX3 regulates the expression of the Gck and glucagon genes. We did not find significant differences in the levels of $P d x 1$ mRNA (Fig. 3a) or PDX1 protein (Fig. $3 \mathrm{~b}$ and g).

We investigated whether the observed islet alterations in Alx3-null mice were accompanied by increased cell death. In newborn wild-type or mutant mice, very few apoptotic cells were found by TUNEL assay (Fig. 4b), and no differences were observed between genotypes (ESM Fig. 2). In contrast, in adult young mice (15 weeks old) Alx3-deficient islets showed a significantly higher percentage of apoptotic cells as compared with wild-type controls (Fig. 4b). In mature mice (36 weeks old) the percentage of apoptotic cells remained practically unchanged in the wildtype group, but in the Alx3-null group apoptotic cells increased significantly as compared with the younger animals, indicating a gradual decline with age (Fig. 4b). This was confirmed by determining the presence of activated caspase-3, which was present throughout the mutant islets (Fig. 4c). To find out whether cell loss was compensated by increased cell replication, we determined the number of islet cells positive for antigen identified by monoclonal antibody Ki 67 (Ki67), but observed no differences between wild-type and Alx3-null mice (ESM Fig. 3).

These experiments indicate that there is a substantial degree of cell loss in the islets of adult Alx3-deficient mice that could have an impact on islet size. To clarify this, we performed morphometric analyses that revealed a reduced 


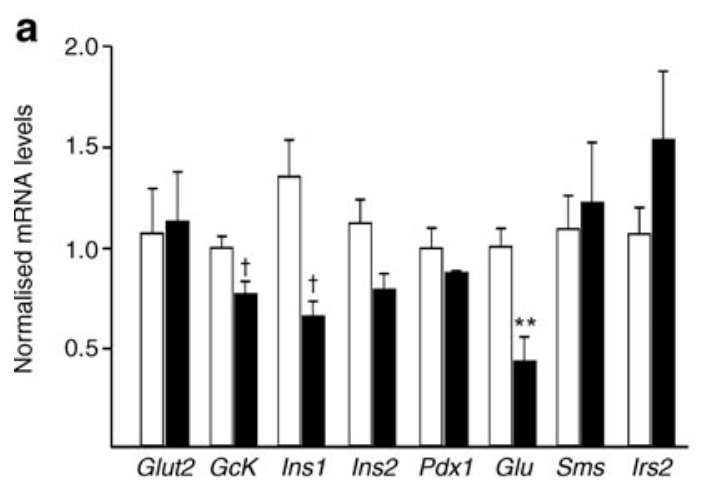

d
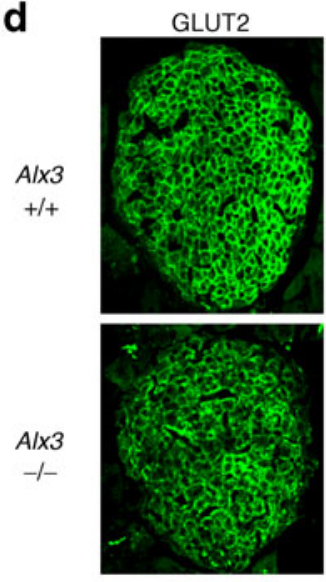

g
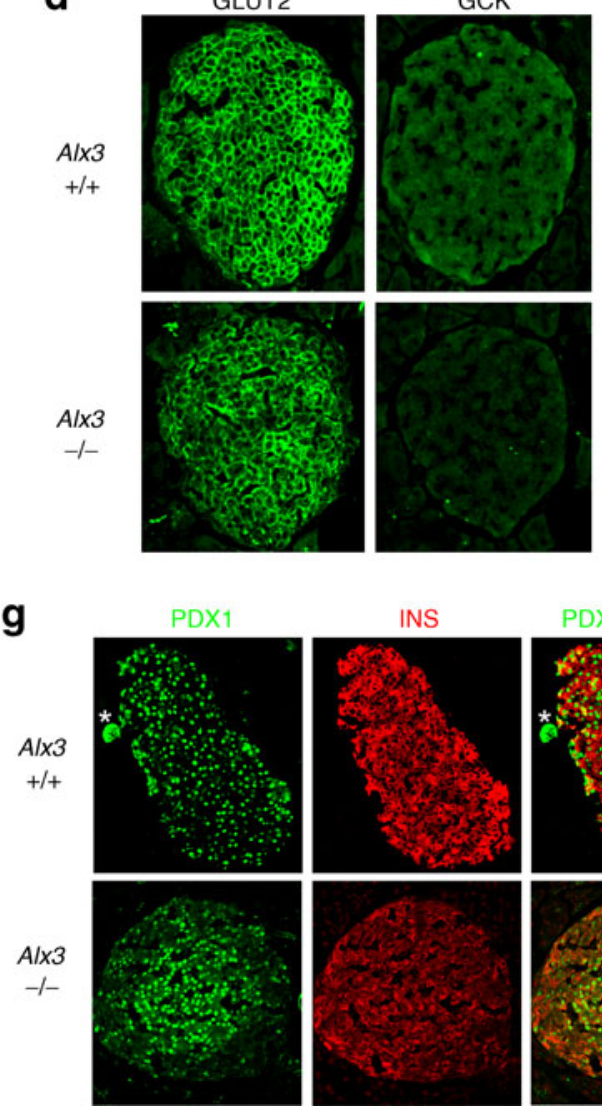

b
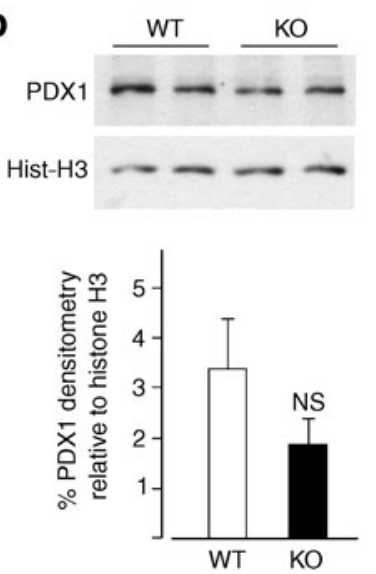
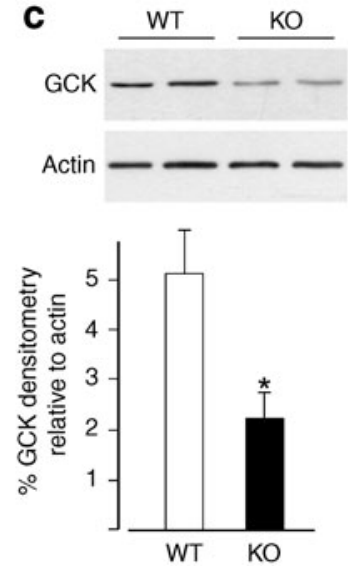

e

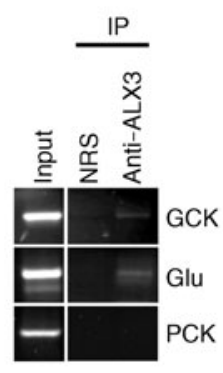

f

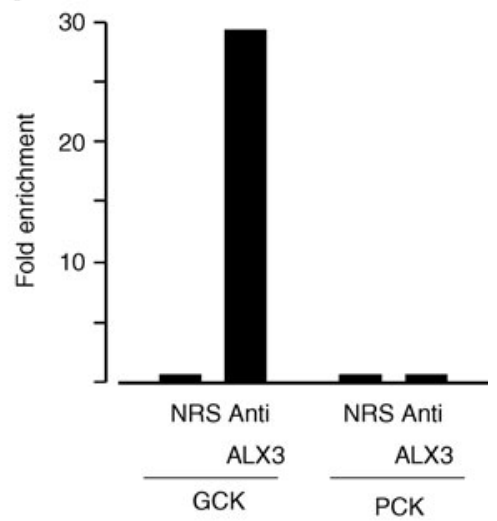

h

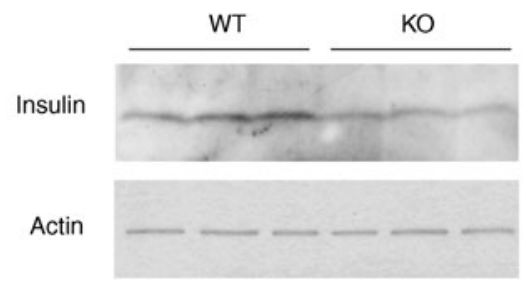

size of Alx3-deficient islets (Fig. 5). The average protein content per islet in mutant mice is reduced by almost half relative to that of controls, reflecting a smaller islet size (Fig. 5d). A comparative analysis of the distribution of islet sizes in histological sections indicated that Alx3-mutant mice lack pancreatic islets of the largest sizes $\left(>70,000 \mu \mathrm{m}^{2}\right.$, Fig. 5f), whereas approximately $10 \%$ of islets in wild-type animals fall within this range. In agreement with these observations, beta cell mass was reduced in the pancreases of Alx3-deficient mice (Fig. 5e).
Age-related insulin resistance in Alx3-deficient mice In the course of the experiments to determine blood insulin concentration after glucose administration described in Fig. 1, we detected one Alx3-deficient mouse within the group of younger animals with an abnormally elevated serum insulin concentration $(1,056.7 \mathrm{pmol} / \mathrm{l}) 2 \mathrm{~h}$ after glucose administration (Fig. 6a; not included in Fig. 1). To explore the possible significance of this finding we increased the number of animals tested for serum insulin at that time point after glucose injection, but no 
4Fig. 3 Alx3-deficient mice exhibit decreased pancreatic islet Gck expression. a Relative levels of mRNA extracted from isolated pancreatic islets of wild-type (white bars) or Alx3-deficient (black bars) mice, as assessed by quantitative RT-PCR. Data represent the mean \pm SEM of 5-10 independent samples amplified in triplicate and normalised to Gapdh. ${ }^{\dagger} p=0.025, * * p<0.01$, Student's $t$ test. Western immunoblots showing levels of PDX1 (b) and GCK (c) carried out with extracts of isolated islets from wild-type (WT) or Alx3-deficient (KO) mice. Histograms represent densitometric analysis performed to quantify the relative intensity of PDX1- and GCK-immunoreactive bands detected by western immunoblot. Results are expressed as a percentage of increment of densitometry measurements of PDX1 or GCK bands (in arbitrary units) relative to the intensity of the corresponding actin or histone H3 bands. Quantification from separate blots ( $n=8$ animals per group) is shown. ${ }^{*} p<0.05$, Student's $t$ test. d Representative examples of sections from the pancreases of wild-type (top) or Alx3-deficient (bottom) mice processed for GLUT2 and GCK immunostaining and analysed by confocal microscopy. e ChIP assay showing PCR amplification of GCK or glucagon chromatin immunoprecipitated with anti-ALX3 antiserum or with control non-immune rabbit serum (NRS) from pancreatic islets isolated from wild-type mice. PCR amplification was not obtained with primers corresponding to the control Pck gene. The panel depicts results from one of two independent experiments yielding similar data. f Results from an additional ChIP experiment analysed by quantitative PCR carried out in triplicate. Data represent fold enrichment relative to input. For each one of the ChIP assays shown in e and $\mathbf{f}$, approximately 1,500 islets from 10-11 animals were used. $\mathbf{g}$ Immunofluorescence images taken from sections of pancreases processed for PDX1 (green) and insulin (red) immunoreactivity and analysed by confocal microscopy. Sections from wild-type and Alx3-null islets mice were strictly processed in parallel for immunostaining, and photographs were taken with identical configuration settings for the confocal microscope and the digital camera. The asterisk denotes a staining artefact. h Western immunoblot showing insulin expression in extracts of isolated islets from three wild-type (WT) or three Alx3-deficient (KO) mice. Samples were resolved using a $4-20 \%$ precast gel (Bio$\mathrm{Rad})$ and a guinea pig anti-insulin antiserum (1:1,000 dilution; Linco Research). GLUT2, glucose transporter 2; Glu, glucagon; Hist, histone; Ins, insulin; IP, chromatin immunoprecipitated; NRS, nonimmune rabbit serum

additional hyperinsulinaemic animals were found within this group (Fig. 6a). In contrast, within the group of 3640 week old animals, from a total of 25 Alx3-null mice tested, $28 \%$ were found to form a distinct subgroup that exhibited significantly increased serum insulin levels (Fig. 6a).

Prompted by this finding, we performed insulin tolerance tests to explore the possibility that peripheral insulin action in Alx3-deficient mice may be impaired due to insulin resistance. We found that insulin administration lowered blood glucose levels in both wild-type and mutant 12-16 week-old mice to a similar extent, indicating that ALX3 deficiency does not impair insulin sensitivity at this age (Fig. 6b). Similarly, in the 36-40 week-old group, we found no statistically significant differences (ANOVA) in insulin action on blood glucose levels between wild-type and most (71.5\%) mutant mice (Fig. 6c). However, in this age group, we found that $28.5 \%$ Alx3-deficient mice were totally unresponsive to insulin (Fig. 6c, dashed line). A similar result was also observed in age-matched females (data not shown). There was no correlation between the presence of insulin insensitivity and weight, which remained within normal values in all animals tested. Taken together, these data indicate that Alx3-deficient mice develop age-dependent insulin resistance.

In an initial effort to investigate the cause of the observed insulin resistance, we monitored insulin-induced phosphorylation of AKT in liver and muscle. We found that insulin administration results in AKT phosphorylation in wild type and non-insulin-resistant $A l x 3$-null mice, but not in insulin-resistant Alx3-mutant mice (Fig. 7), indicating that peripheral insulin signalling is compromised in these animals.

\section{Discussion}

Our work indicates that the main consequences of $A l x 3$ deficiency in pancreatic islets are decreased content of insulin and GCK, increased apoptosis and reduced islet size. The effect on insulin is consistent with our previous findings that ALX3 regulates insulin gene expression [19]. As a consequence of these defects, the amount of insulin released in response to glucose stimulation in vitro is comparatively reduced. Importantly, this reduction does not necessarily indicate a defect in the insulin secretory machinery per se, a notion supported by the observation that levels of circulating insulin in vivo were not found to be reduced in fasting Alx3-deficient mice in basal conditions or after a glucose challenge.

One possible interpretation of these apparently contrasting results is that the mutant islets are overstimulated by the hyperglycaemic environment in vivo to maintain blood insulin levels close to normal values in the presence of elevated glucose levels [24]. In addition, the observation that $A l x 3$-deficient mice exhibit relatively high blood glucose levels in fasting conditions despite normal levels of serum insulin is consistent with the existence of a defect in the glucose-sensing mechanisms in beta cells, resulting in a displacement of the threshold at which glucose concentration elicits the release of appropriate amounts of insulin to the circulation. As GCK acts as a glucose sensor in beta cells, this notion is in line with our finding of reduced GCK levels in Alx3-null mice $[25,26]$.

The reduced content of GCK associated with ALX3 deficiency is relevant because loss-of-function mutations in $G C K$ in humans are a known cause of MODY2. Similarly to Alx3-deficient mice, these patients exhibit chronic mild 


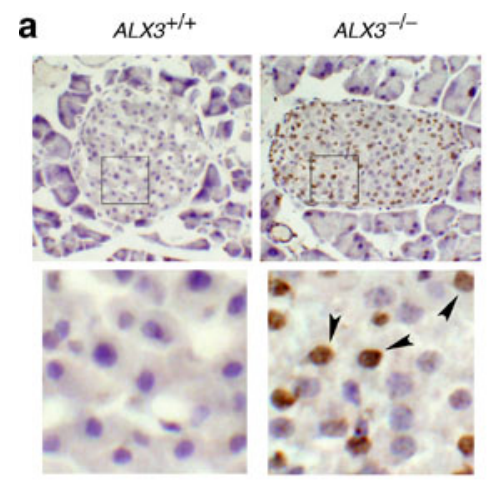

b

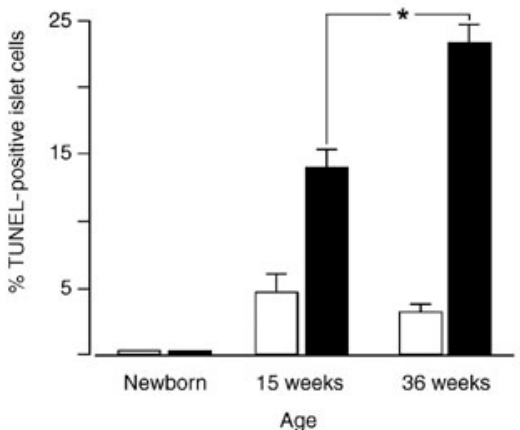

Fig. 4 Alx3-deficient islets exhibit increased apoptosis. a Images of islets from sections of pancreases from 36 week old wild-type and Alx3-deficient mice processed for TUNEL staining and counterstained with haematoxylin. Insets indicated on each section are shown at higher magnification on the bottom panels. Examples of TUNELpositive nuclei are indicated by arrowheads. b Quantification of the percentage of TUNEL-positive cells in islets of wild-type (white bars) or Alx3-mutant (black bars) mice of different ages. For newborn mice, digital images (see ESM Fig. 2) from two wild-type

fasting hyperglycaemia in the presence of normal levels of serum insulin, reflecting a resetting of their homeostatic control such that increased glucose levels are necessary to elicit normal levels of insulin secretion [27-29]. Mouse models of MODY2 by heterozygous disruption of the Gck gene are also characterised by mild fasting hyperglycaemia and impaired glucose tolerance [25, 30]. Thus, ALX3 deficiency could primarily affect islet cell function by a combined effect on both insulin and GCK production, as our data indicate that the Gck gene, as well as the insulin gene [19], is a direct target regulated by ALX3 at the transcriptional level.

Another important feature of Alx 3 -deficient islets is the relatively high number of apoptotic cells in adult, but not in newborn mice, indicating that ALX3 is important for the long-term maintenance of islet cell survival. Similar to ALX3, deficiency of other transcription factors that regulate insulin gene expression and beta cell function also leads to increased beta cell apoptosis and impaired islet function [4]. Increased apoptosis in pancreatic islets can lead to

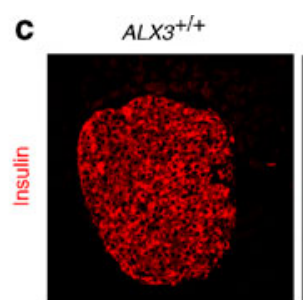

$A L \times 3^{-1-}$
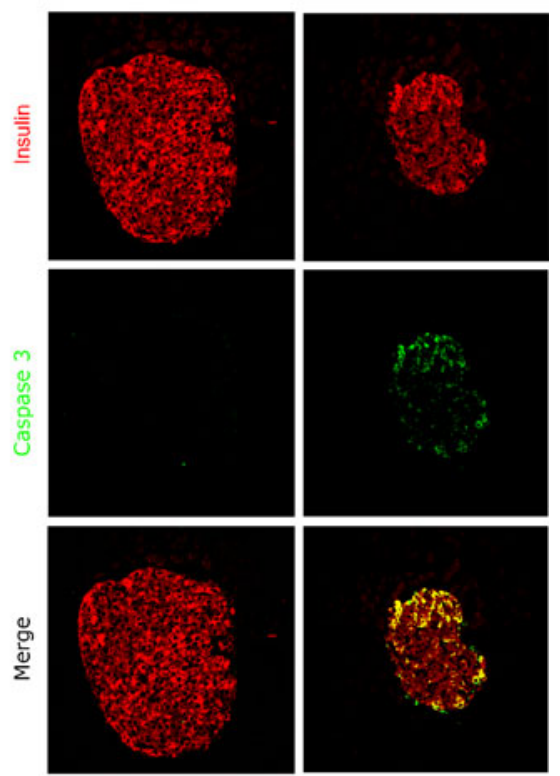

and three Alx3-null mice (33-40 sections per genotype) were used for quantification. For adult mice, a total of 31-50 islets from two different mice in each genotype and age were used. Data represent the mean \pm SEM. ${ }^{*} p<0.0001$, Student's $t$ test. c Immunofluorescence images taken from sections of pancreases processed for insulin (red) and activated caspase-3 (green) immunoreactivity. Note that activated caspase-3 immunoreactivity is only evident in the Alx3-mutant islet

decreased beta cell mass to the point of compromising islet cell function $[6,31,32]$.

The mechanisms by which lack of ALX3 results in islet cell apoptosis are currently unknown. Decreased GCK activity can lead to increased apoptosis in islets [3, 33]. Indeed, GCK has been shown to be associated with the proapoptotic protein BCL2-associated agonist of cell death (BAD), which acts as a molecular switch regulating metabolic activity and apoptosis in beta cells [34]. As content of GCK is reduced in pancreatic islets of Alx3-deficient mice, this may well provide a mechanistic explanation for the increased rate of cell death observed in these mice. Interestingly, beta cell-specific disruption of the Gck gene may lead to altered islet cell distribution [30]. In Alx3-deficient mice we observed altered islet architecture, evidenced by mislocalisation of a number of alpha and delta cells found scattered throughout the islet core (not shown). Defects in islet architecture have often been associated with increased beta cell apoptosis $[6,31]$. 

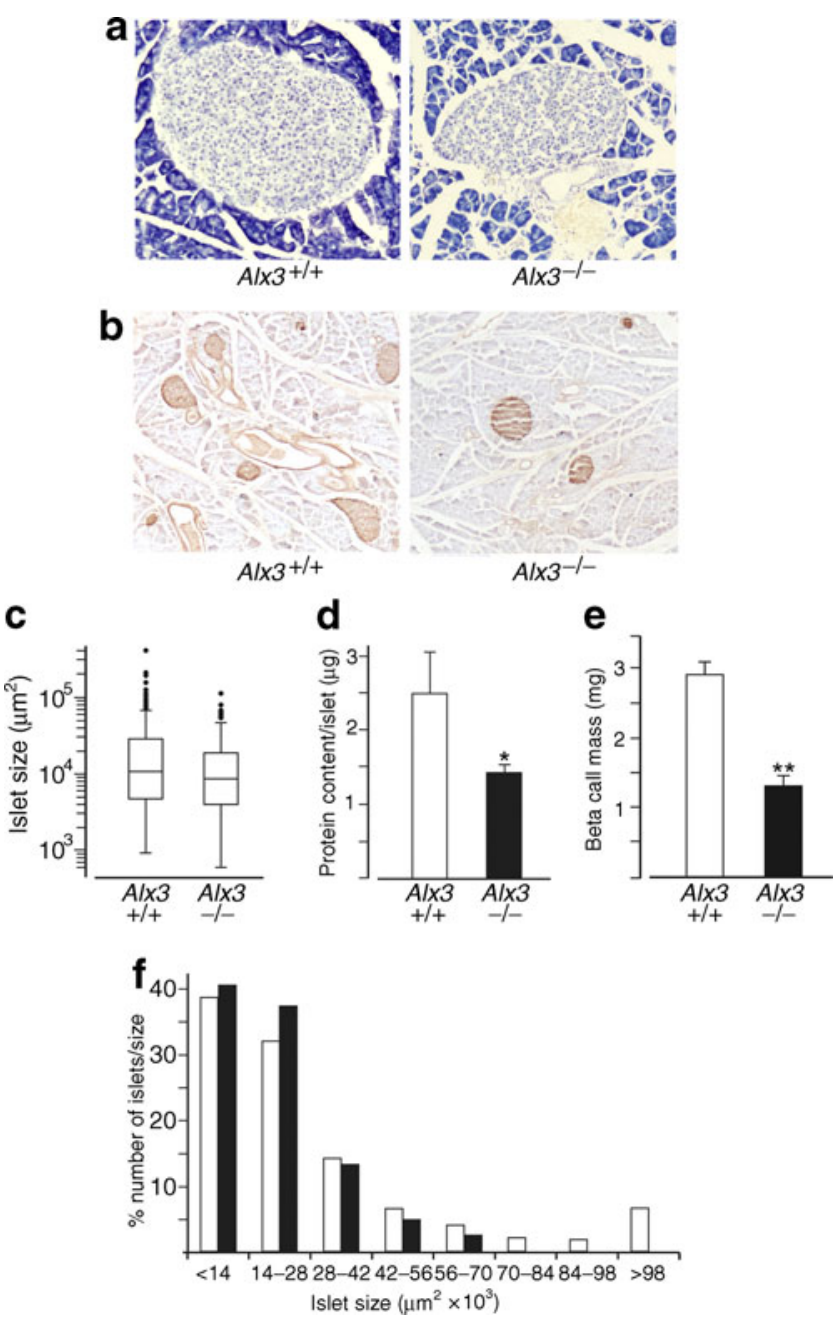

Fig. 5 Reduced size of pancreatic islets in Alx3-deficient mice. a Representative examples of Cresyl Violet-stained sections of pancreases used for morphometric analyses. b Representative examples of insulin immunostained sections of pancreases used for calculation of the beta cell mass. c Islet size determined in sections similar to those shown in a. A total of 337 islets from three wild-type animals and 309 islets from three Alx3-deficient mice were counted. Data are represented as box-and-whisker plots showing median values, first and third quartiles, as well as outliers. Statistical analysis indicated that differences between wild-type and $A l \times 3$-deficient islets are significant $(p<0.01$; Mann-Whitney $U$ test). d Relative protein content per islet as determined by Bradford assays of lysates of groups of isolated islets (70-130 islets per lysate) from five wild-type and eight $A l x 3$-deficient mice. ${ }^{*} p<0.05$, Student's $t$ test. e Beta cell mass calculated from sections of pancreases immunostained for insulin. ${ }^{* *} p<0.0001$, Student's $t$ test. Data in $\mathbf{c}$ and $\mathbf{d}$ are mean \pm SEM f Distribution of islets according to size. White bars, wild-type mice; black bars, Alx3-deficient mice. Note the absence of islets of the largest sizes $\left(>70 \mu \mathrm{m}^{2} \times 10^{3}\right)$ in the pancreases from Alx3-null mice. Statistical analysis indicated that differences between the distribution of wild-type and $A l x 3$-deficient islets are significant ( $p<0.001, \chi^{2}$ test)

Nonetheless, lack of ALX3 may lead to apoptosis by other mechanisms that are unrelated to GCK production. In support of this notion, we have observed an increased rate of apoptotic cell death associated with

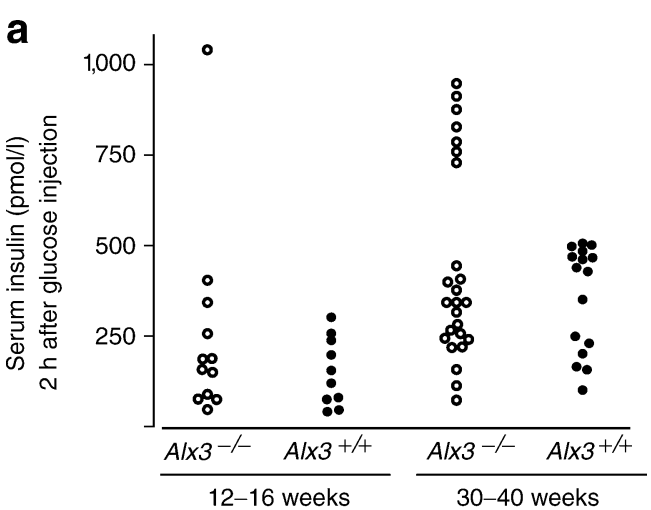

b
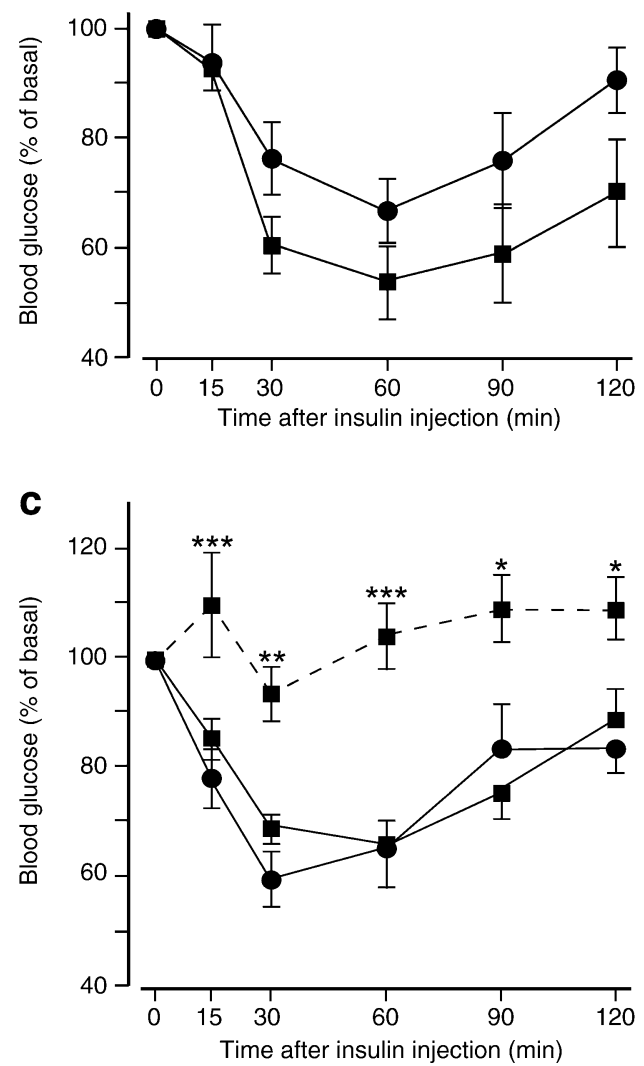

Fig. 6 Alx3-deficient mice develop age-dependent insulin resistance. a Serum insulin levels of individual wild-type or Alx3-deficient mice of two different age groups, detected $2 \mathrm{~h}$ after an intraperitoneal injection of glucose ( $2 \mathrm{~g} / \mathrm{kg}$ body weight), administered after an overnight fasting period. Note the presence of only one high value for serum insulin in the Alx3-mutant 12-16 week old group, and several (28\%) in the Alx3mutant 36-40 week old mutant mice. b, c Insulin tolerance tests showing relative changes in blood glucose concentrations observed in wild-type (circles) or Alx3-deficient (squares) mice after intraperitoneal administration of insulin. Data from animals of 12-16 weeks of age (b) or 36-40 weeks of age (c) are shown, and represent the mean \pm SEM. In b, six wild-type and seven mutant mice were used. In c, the data from seven wild-type animals were used to plot the graph, but 11 additional wild-type mice were tested yielding similar results. In the 36-40 week age group, a total of 21 Alx3-mutant mice were tested. In six of these, blood glucose levels were not decreased by the administration of insulin, represented in the graph by a dashed line. ${ }^{*} p<0.05,{ }^{*} p<0.01$ and $* * * p<0.001$ as compared with wild-type mice (ANOVA) 

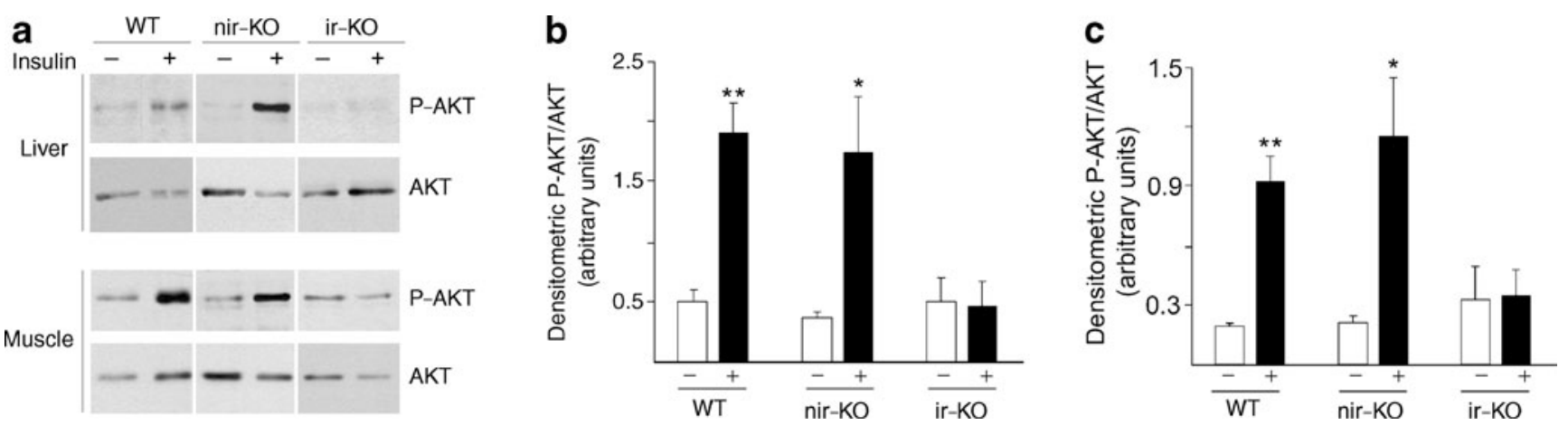

Fig. 7 Alx3-deficient mice develop impaired insulin signalling in peripheral tissues. a Western immunoblots showing phosphorylated (P-) and total AKT in liver or muscle extracts from wild-type (WT) or Alx3-deficient non-insulin-resistant (nir-KO) or insulin resistance (ir-KO) mice. Each lane represents extracts from the same animal. $\mathbf{b}, \mathbf{c}$ Results of densitometric measurements for quantification of the intensity of P-AKT bands relative to that of total AKT bands in liver (b) and muscle (c). Black bars, animals treated with insulin $(0.75 \mathrm{U} / \mathrm{kg}$ i.p. $)$; white bars, control non-treated mice. Three mice were used for each condition. Data represent mean \pm SEM. ${ }^{*} p<0.05,{ }^{* *} p<0.01$, Student's $t$ test
Alx3 deficiency in the cranial mesenchyme of developing embryos [35], a major site of Alx3 expression during development [18].

The smaller size observed in Alx3-deficient pancreatic islets may be related to their relatively high proportion of apoptotic cells, and may contribute to impaired islet cell function. This condition may be important even though Alx3-deficient mice do not appear to develop high hyperglycaemic values typical of overt diabetes, because if increased cell death by apoptosis remains a dominant determinant in Alx3-deficient islets, compensation by hyperplasia in response to diabetes-related risk factors such as a high fat diet could be compromised. A similar situation has been recently observed in $P d x l$ haploinsufficient mice [32]. We did not find evidence of increased proliferation in islets to compensate for the apoptotic cell loss. Thus, Alx 3 deficiency could potentially constitute a vulnerability factor for the development of diabetes in response to a particular challenge such as an unbalanced diet, an important question that deserves further investigation.

In young (12-16 weeks old) Alx3-deficient mice, the existence of impaired glucose tolerance in the presence of serum insulin levels that were similar to those found in control animals was initially suggestive of insulin resistance. However, all animals of this age tested for insulin tolerance showed normal insulin sensitivity, hence our interpretation, discussed above, that the primary defect in these animals relates most likely to the islets, and not to the existence of peripheral insulin resistance. In contrast, the observations of hyperglycaemia in the presence of increased serum insulin levels, impaired insulin tolerance tests and defective insulin signalling in a significant number of Alx3-deficient mice within the 36-40-week-old group, clearly indicates the development of insulin resistance in an age-dependent manner. It is not clear why only a proportion of mice develop insulin resistance, but this could simply reflect the progressive nature of this condition. Thus, it remains to be determined whether the incidence of insulin resistance in even older Alx3-deficient mice is higher than that found in the present study. Conversely, if Alx3-deficient animals exhibit increased susceptibility to environmental factors, it is possible that an appropriate challenge such as high-fat diet could result in increased proportion of insulin resistance, even in younger animals.

Although insulin-resistant Alx3-null mice have impaired insulin signalling evidenced by decreased AKT phosphorylation in liver and muscle, the ultimate mechanism responsible for this defect is unknown. Insulin resistance can develop in animals with reduced islet cell mass [36, 37], an observation in line with the notion that metabolic alterations in diabetes are the consequence of a primary defect in beta cells [38-40]. In any case, it is possible that the observed phenotype reflects beta cell defects in combination with insulin resistance.

In summary, our data indicate that Alx3 deficiency alters islet cell function and compromises islet cell viability, leading to an impairment of glucose homeostasis. Although the alterations observed in this study do not reach the degree of severity typical of overt diabetes, it is known that certain mutations, or even common variants, of some of the genes that encode transcription factors involved in the maintenance of islet cell function may increase the risk to develop diabetes [32, 41-43]. Of note, the human $A L X 3$ gene is located in chromosome $1 \mathrm{p} 21$ p13, a region synthenic with regions of rat chromosome 2 and mouse chromosome 3 that have been linked to diabetes by quantitative trait loci analysis [44-46]. Therefore, our data support the proposal that $A L X 3$ is a putative candidate gene to take into account for predisposition to diabetes. 
Acknowledgements We are very grateful to F. Meijlink (Netherlands Institute for Developmental Biology, Utrecht, the Netherlands) for providing Alx3-deficient mice, J. Habener (Massachusetts General Hospital, Boston, MA, USA) for the anti-PDX1 antiserum, and A. M. Valverde and A. González-Rodriguez (Instituto de Investigaciones Biomédicas 'Alberto Sols', CSIC/UAM and CIBERDEM, Madrid, Spain) for providing the anti-AKT antiserum, and for advice on experiments with liver extracts. This work was funded by the Spanish Ministry of Science and Innovation (BFU2008-01283) and by the Instituto de Salud Carlos III. CIBERDEM is an initiative of the Instituto de Salud Carlos III.

Duality of interest The authors declare that there is no duality of interest associated with this manuscript.

\section{References}

1. Song B, Scheuner D, Ron D, Pennathur S, Kaufman RJ (2008) Chop deletion reduces oxidative stress, improves beta cell function, and promotes cell survival in multiple mouse models of diabetes. J Clin Invest 118:3378-3389

2. Hashimoto N, Kido Y, Uchida T et al (2006) Ablation of PDK1 in pancreatic $\beta$ cells induces diabetes as a result of loss of $\beta$ cell mass. Nature Genet 38:589-593

3. Lee JW, Kim WH, Lim JH et al (2009) Mitochondrial dysfunction: glucokinase downregulation lowers interaction of glucokinase with mitochondria, resulting in apoptosis of pancreatic $\beta$-cells. Cell Signal 21:69-78

4. Bernardo AS, Hay CW, Docherty K (2008) Pancreatic transcription factors and their role in the birth, life and survival of the pancreatic $\beta$ cell. Mol Cell Endocrinol 294:1-9

5. Naya FJ, Huang HP, Qiu Y et al (1997) Diabetes, defective pancreatic morphogenesis, and abnormal enteroendocrine differentiation in BETA2/NeuroD-deficient mice. Genes Dev 11:2323-2334

6. Johnson JD, Ahmed NT, Luciani DS et al (2003) Increased islet apoptosis in $\mathrm{Pdx}^{+/-}$mice. J Clin Invest 111:1147-1160

7. Du A, Hunter CS, Murray J et al (2009) Islet-1 is required for the maturation, proliferation and survival of the endocrine pancreas. Diabetes. doi:10.2337/db08-0987

8. Wobser H, Dussmann H, Kogel D et al (2002) Dominant-negative suppression of HNF-1 alpha results in mitochondrial dysfunction, INS-1 cell apoptosis, and increased sensitivity to ceramide-, but not glucose-induced cell death. J Biol Chem 277:6413-6421

9. Malecki MT, Jhala U, Antonellis A et al (1999) Mutations in NEUROD1 are associated with the development of type 2 diabetes. Nat Genet 23:323-328

10. Stoffers DA, Ferrer J, Clarke WL, Habener JF (1997) Early-onset type-II diabetes mellitus (MODY4) linked to IPF1. Nature Genet 17:138-141

11. Hani EH, Stofers DA, Chevre JC et al (1999) Defective mutations in the insulin promoter factor-1 (IPF-1) gene in late-onset type 2 diabetes mellitus. J Clin Invest 104:41-48

12. Barat-Houari M, Clement K, Vatin V et al (2002) Positional candidate gene analysis of Lim homeobox gene (Isl-1) on chroosome $5 \mathrm{q} 11-\mathrm{q} 13$ in a French morbidly obese population suggests indication for association with type 2 diabetes. Diabetes 51:1640-1643

13. Shimomura H, Sanke T, Hanabusa T, Tsunoka K, Furuta H, Nanjo K (2000) Nonsense mutation of islet-1 gene (Q310X) found in a type 2 diabetic patient with a strong family history. Diabetes 49:1597-1600

14. Yamagata K, Oda N, Kaisaki PJ et al (1996) Mutations in the hepatocyte nuclear factor-1 alpha gene in maturity-onset diabetes of the young (MODY3). Nature 384:455-458
15. Servitja JM, Pignatelli M, Maestro MA et al (2009) Hnfl $\alpha$ (MODY3) controls tissue-specific transcriptional programs and exerts opposed effects on cell growth in pancreatic islets and liver. Mol Cell Biol 29:2945-2959

16. Oliver-Krasinski JM, Kasner MT, Yang J et al (2009) The diabetes gene $\mathrm{Pdx} 1$ regulates the transcriptional network of pancreatic endocrine progenitor cells in mice. J Clin Invest 119:1888-1898

17. Servitja JM, Ferrer J (2004) Transcriptional networks controlling pancreatic development and beta cell function. Diabetologia 47:597-613

18. Ten Berge D, Brouwer A, El Bahi S, Guenet JL, Robert B, Meijlink F (1998) Mouse Alx3: an aristaless-like homeobox gene expressed during embryogenesis in ectomesenchyme and lateral plate mesoderm. Dev Biol 199:11-25

19. Mirasierra M, Vallejo M (2006) The homeoprotein Alx3 expressed in pancreatic $\beta$-cells regulates insulin gene transcription by interacting with the basic helix-loop-helix protein E47. Mol Endocrinol 20:2876-2889

20. Beverdam A, Brouwer A, Reijnen M, Korving J, Meijlink F (2001) Severe nasal clefting and abnormal embryonic apoptosis in Alx3/Alx4 double mutant mice. Development 128:39753986

21. González-Rodríguez A, Escribano O, Alba J, Rondinone CM, Benito M, Valverde AM (2007) Levels of protein tyrosine phosphatase 1B determine susceptibility to apoptosis in serumdeprived hepatocytes. J Cell Physiol 212:76-88

22. Moates JM, Nanda S, Cissell MA, Tsai MJ, Stein R (2003) BETA2 activates transcription from the upstream glucokinase gene promoter in islet $\beta$-cells and gut endocrine cells. Diabetes 52:403-408

23. Artner I, Hang Y, Guo M, Gu G, Stein R (2008) MafA is a dedicated activator of the insulin gene in vivo. $\mathrm{J}$ Endocrinol 198:271-279

24. Andrali SS, Sampley ML, Vanderford NL, Ozcan S (2008) Glucose regulation of insulin gene expression in pancreatic $\beta$ cells. Biochem J 415:1-10

25. Postic C, Shiota M, Magnuson MA (2001) Cell-specific roles of glucokinase in glucose homeostasis. Recent Prog Horm Res $56: 195-217$

26. Zelent D, Najafi H, Odili S et al (2005) Glucokinase and glucose homeostasis: proven concepts and new ideas. Biochem Soc Trans 33:306-310

27. Byrne MM, Sturis J, Clement K et al (1994) Insulin secretory abnormalities in subjects with hyperglycemia due to glucokinase mutations. J Clin Invest 93:1120-1130

28. Pearson ER, Velho G, Clark P et al (2001) $\beta$-Cell genes and diabetes: quantitative and qualitative differences in the pathophysiology of hepatic nuclear factor- $1 \alpha$ and glucokinase mutations. Diabetes 50:S101-S107

29. Velho G, Blanch H, Vaxillaire M et al (1997) Identification of 14 new glucokinase mutations and description of the clinical profile of 42 MODY-2 families. Diabetologia 40:217-224

30. Terauchi Y, Sakura H, Yasuda K et al (1995) Pancreatic $\beta$-cellspecific targeted disruption of glucokinase gene. Diabetes mellitus due to defective insulin secretion to glucose. J Biol Chem 270:30253-30256

31. Johnson JD, Ford EL, Bernal-Mizrachi E et al (2006) Suppressed insulin signaling and increased apoptosis in $\mathrm{Cd} 38$-null islets. Diabetes 55:2737-2746

32. Sachdeva MM, Claiborn KC, Khoo C et al (2009) Pdx1 (MODY4) regulates pancreatic beta cell susceptibility to ER stress. Proc Natl Acad Sci USA 106:19090-19095

33. Kim WH, Lee JW, Suh YH et al (2005) Exposure to chronic high glucose induces $\beta$-cell apoptosis through decreased interaction of glucokinase with mitochondria. Downregulation of glucokinase in pancreatic $\beta$-cells. Diabetes 54:2602-2611 
34. Danial NN, Walensky LD, Zhang CY et al (2008) Dual role of proapoptotic BAD in insulin secretion and beta cell survival. Nat Med 14:144-153

35. Lakhwani S, Garcia-Sanz P, Vallejo M (2010) Alx3-deficient mice exhibit folic acid-resistant craniofacial midline and neural tube closure defects. Dev Biol 344:869-880

36. Matveyenko AV, Butler PC (2006) Beta cell deficit due to increased apoptosis in the human islet amyloid polypeptide transgenic (HIP) rat recapitulates the metabolic defects present in type 2 diabetes. Diabetes 55:2106-2114

37. Matveyenko AV, Veldhuis JD, Butler PC (2006) Mechanisms of impaired fasting glucose and glucose intolerance induced by $50 \%$ pancreatectomy. Diabetes 55:2347-2356

38. Kahn SE, Hull RL, Utzschmeider KM (2006) Mechanisms linking obesity to insulin resistance and type 2 diabetes. Nature 444:840846

39. Matveyenko AV, Butler PC (2008) Relationship between $\beta$-cell mass and diabetes onset. Diabetes Obes Metab 10:23-31

40. Prentki M, Nolan CJ (2006) Islet $\beta$ cell failure in type 2 diabetes. J Clin Invest 116:1802-1812
41. Holmkvist J, Almgren P, Lyssenko V et al (2008) Common variants in maturity-onset diabetes of the young genes and future risk of type 2 diabetes. Diabetes 57:1738-1744

42. Shih DQ, Heimesaat M, Kuwajima S, Stein R, Wright C, Stoffel $M$ (2002) Profound defects in pancreatic $\beta$-cell function in mice with combined heterozygous mutations in $\mathrm{Pdx}-1$, Hnf- $1 \alpha$, and Hnf-3ß. Proc Natl Acad Sci USA 99:3818-3823

43. Neve B, Fernandez-Zapico ME, Ashkenazi-Katalan V et al (2005) Role of transcription factor KLF11 and its diabetes-associated gene variants in pancreatic beta cell function. Proc Natl Acad Sci USA 102:4807-4812

44. Wallace KJ, Wallis RH, Collins SC et al (2004) Quantitative trait locus dissection in congenic strains of the Goto-Kakizaki rat identifies a region conserved with diabetes loci in human chromosome 1q. Physiol Genomics 19:1-10

45. Wallis RH, Wang K, Marandi L et al (2009) Type 1 diabetes in the BB rat: a polygenic disease. Diabetes 58:1007-1017

46. Gao P, Jiao Y, Xiong Q, Wang CY, Gerling I, Gu W (2008) Genetic and molecular basis of QTL of diabetes in mouse: genes and polymorphisms. Curr Genomics 9:324-337 